\title{
Post-mortem findings in mood disorders, nightmares and therapeutic approaches of psychiatric diseases
}

\author{
Andrea Schmitt • Peter Falkai
}

Published online: 6 October 2012

(c) Springer-Verlag Berlin Heidelberg 2012

Dedicated to post-mortem studies on mood disorders in its first part, this edition opens with Bernstein et al. [1] comparing the pathomorphology of mammillary bodies in patients with major depression disorder (MDD) and bipolar I disorder (BD) to control subjects. Via assessing the mammillary body and fornix volumes, number of neurons and neuronal densities, the group found male control subjects to have significantly larger mammillary bodies compared with females. They furthermore detected a significant reduction of the left mammillary body volume in BD patients compared with those with MDD. Another study of this work group [2] focused on alterations in GABAergic neurotransmission which are assumed to play a crucial role in the pathophysiology of mood disorders. The authors found an accentuated increase in GAD-ir neuropil density in the entorhinal cortex and the hippocampus of MDD compared with BD patients and controls. Another interesting finding was the dorsolateral prefrontal cortex in BD to be the only area showing a significant decrease of GADir neuropil density compared with MDD and controls. The authors suggest a diathesis of the GABAergic system in mood disorders as possibly helpful in differentiating the pathophysiology of MDD from BD. Although protein phosphorylation, involved in specific processes like cellular signaling, might add to unravel the molecular substructure of MDD, psychiatric phosphoproteome studies are rare. Martins-de-Souza et al. [3] aimed at filling this gap with a comparative phosphoproteome analysis of dorsolateral prefrontal cortex tissues in major depression

A. Schmitt $(\bowtie) \cdot$ P. Falkai

Department of Psychiatry and Psychotherapy,

Ludwig-Maximilians-University Munich,

Nußbaumstr. 7, 80336 Munich, Germany

e-mail: Andrea.Schmitt@med.uni-muenchen.de using liquid chromatography mass spectrometry. They could identify more than 5,000 phosphopeptides, corresponding to ca. 800 non-redundant proteins. Compared with controls, 90 of these proteins showed differential levels of phosphorylation in MDD patients, most of them associated with synaptic transmission and cellular architecture. This might point out potential biomarker candidates and thus add to comprehend the pathophysiology of MDD.

Due to preliminary evidence from pharmacological studies on verbal and visuospatial subcomponents of working memory (WM) being subject to differential neurotransmitter modulation, Zilles et al. [4] genotyped 20 healthy controls and 80 patients with schizophrenia, BD, or obsessive-compulsive disorder with respect to the dopaminergic and serotonergic systems. While DAT genotype revealed a significant effect on visuospatial WM, and 5-HTT again a significant one on verbal WM task performance, COMT did not show an influence on either domain. This evidently differential impact of genetic polymorphisms on verbal and visuospatial WM functioning adds to prior suppositions about the existence of schizophrenia subgroups with patients exhibiting isolated deficits in only one WM domain and supports the idea of endophenotypically and pathophysiologically distinct patient subgroups needing personalized therapeutic approaches.

While behavioral and neuroimaging studies have shown an association of distinct symptom clusters like impulsivity with orbitofrontal cortex (OFC) dysfunction in patients with borderline personality disorder, neither the presence of abnormal OFC activity patterns during resting-state conditions nor a possible specific association of OFC dysfunction with impulsivity has been investigated so far. Accordingly, in an MRI study, Wolf et al. [5] found a significant positive relationship between medial and lateral 
orbitofrontal flood flow and impulsivity scores but not with dissociation tension and depression. Thus, they conclude that dysfunction in medial and lateral OFC regions could specifically mediate impulsivity symptoms.

There exist predominantly questionnaire-based studies revealing a relation between nightmares and impaired sleep quality. Simor et al. [6] used polysomnographic recordings to investigate the sleep architecture of 17 individuals with frequent nightmares compared to 23 controls and found reduced sleep efficiency, increased wakefulness, reduced amount of slow wave sleep plus increased nocturnal awakenings, especially from Stage 2 sleep, in nightmare probands. They furthermore exhibited longer durations of REM sleep due to heightened negative affect. These results support a relationship between nightmares and altered sleep architecture.

In a pharmacokinetic study with the wake-promoting drug modafinil, which has been suggested as novel treatment for several psychiatric disorders like ADHD and depression, Hellman-Regen et al. [7] investigated the role of the cytochrome P450 and drug interactions. They found modafinil to induce hepatic CYP enzymes and increase CYP3A4 expression in human neuroblastoma cells. This upregulation was associated with increased retinoic acid degradation which in turn during treatment resulted in decreased neuronal differentiation. Either effect could be blocked by specific CYP3A4 inhibitor erythromycin. Treatment of a murine glioma cell line with modafinil also resulted in increased proliferative activity associated with upregulation of retinoic acid-degrading CYP26A1. The authors assume that psychopharmacological agents like modafinil may directly act on CYP enzymes. Ginsberg et al. [8] conducted a follow-up on an own trial with OROS-methylphenidate in 30 adult male prison inmates with ADHD to evaluate long-term effectiveness and maintenance of improvement on cognition, motor activity, institutional behavior and quality of life. During this 47-week open-label extension accompanied by psychosocial treatment programs, the authors noted significant improvement of verbal and visuospatial WM and abstract verbal reasoning. Additionally, the quality of life domains partly showed a significant relationship to improvements in attention.

This edition closes with Christian Baicus' [9] objection against a study on obesity possibly being an independent risk factor for coronary heart disease in patients without metabolic syndrome treated with second-generation antipsychotics [10]. He states that the authors did not take into account different risks in obese and nonobese patients and insinuates that the authors wanted to exclude obesity as an independent risk factor because they stratified by it. $\mathrm{He}$ furthermore criticizes the exclusion of patients with metabolic syndrome as well as those taking antihyperintensive, hypoglycemic and lipid-lowering drugs which does not allow a differentiation between obese and nonobese patients.

\section{References}

1. Bernstein H-G, Klix M, Dobrowolny H, Brisch R, Steiner J, Bielau H, Gos T, Bogerts B (2012) A postmortem assessment of mammillary body volume, neuronal number and densities, and fornix volume in subjects with mood disorders. Eur Arch Psychiatry Clin Neurosci. doi:10.1007/s00406-012-0300-4

2. Gos T, Steiner J, Bielau H, Dobrowolny H, Günther K, Mawrin C, Krzyżanowski M, Hauser R, Brisch R, Bernstein H-G, Jankowski Z, Braun K, Bogerts B (2012) Differences between unipolar and bipolar I depression in the quantitative analysis of glutamic acid decarboxylase-immunoreactive neuropil. Eur Arch Psychiatry Clin Neurosci. doi:10.1007/s00406-012-0315-x

3. Martins-de-Souza D, Guest PC, Vanattou-Saifoudine N, Rahmoune H, Bahn S (2012) Phosphoproteomic differences in major depressive disorder postmortem brains indicate effects on synaptic function. Eur Arch Psychiatry Clin Neurosci. doi:10.1007/s 00406-012-0301-3

4. Zilles D, Meyer J, Schneider-Axmann T, Ekawardhani S, Gruber E, Falkai P, Gruber O (2012) Genetic polymorphisms of 5-HTT and DAT but not COMT differentially affect verbal and visuospatial working memory functioning. Eur Arch Psychiatry Clin Neurosci. doi:10.1007/s00406-012-0312-0

5. Wolf RC, Thomann PA, Sambataro F, Vasic N, Schmid M, Wolf ND (2012) Orbitofrontal cortex and impulsivity in borderline personality disorder: an MRI study of baseline brain perfusion. Eur Arch Psychiatry Clin Neurosci. doi:10.1007/s00406-012-0 303-1

6. Simor P, Horváth K, Gombos F, Takács KP, Bódizs R (2012) Disturbed dreaming and sleep quality: altered sleep architecture in subjects with frequent nightmares. Eur Arch Psychiatry Clin Neurosci. doi:10.1007/s00406-012-0318-7

7. Hellmann-Regen J, Gertz K, Uhlemann R, Colla M, Endres M, Kronenberg G (2012) Retinoic acid as target for local pharmacokinetic interaction with modafinil in neural cells. Eur Arch Psychiatry Clin Neurosci. doi:10.1007/s00406-012-0309-8

8. Ginsberg Y, Hirvikoski T, Grann M, Lindefors N (2012) Longterm functional outcome in adult prison inmates with ADHD receiving OROS-methylphenidate. Eur Arch Psychiatry Clin Neurosci. doi:10.1007/s00406-012-0317-8

9. Baicus C (2012) A study that shows nothing. Eur Arch Psychiatry Clin Neurosci. doi:10.1007/s00406-012-0310-2

10. Correll CU, Kane JM, Manu P (2011) Obesity and coronary risk in patients treated with second-generation antipsychotics. Eur Arch Psychiatry Clin Neurosci 261:417-423 